\title{
ASSESSMENT OF ANTIBACTERIAL EFFICACY OF DIFFERENT OBTURATION MATERIALS FOR PRIMARY TEETH (AN IN VITRO STUDY)
}

\author{
Hala Shaban Ibrahim*, Nagwa Mohammed Khattab ${ }^{* *}$ and Mina Kamal Yassa***
}

\begin{abstract}
Background: A desirable property of an obturation material in primary teeth is its antibacterial effect, this property promotes the disinfection of the pulp canals and improve the prognosis of pulpectomy and treatment outcomes.

Aim of the study: evaluation the antibacterial potential of various obturation materials for primary teeth; Zinc oxide eugenol (ZOE) only, calcium hydroxide with iodoform (Metapex) and a mixture of zinc oxide eugenol, calcium hydroxide and iodoform (Endoflas) against selected strains of bacteria; Enterococcus faecalis and Bacteroid fragilis.
\end{abstract}

Materials and Methods: Agar diffusion method was used in which the antibacterial potential was tested by measuring the inhibition zone around each tested obturating material.

Results: (Endoflas) showed the highest mean value of inhibition zone, in both types of bacteria, over the (Zinc-oxide and Eugenol) and (Metapex). The difference between the three obturating materials was statistically significant.

Conclusion: The three obturating materials reported different antibacterial effect however, Endoflas was superior to Metapex and ZOE.

KEY WORDS: obturating materials, Pulpectomy, Endoflas, calcium hydroxide with iodoform.

\section{INTRODUCTION}

Primary teeth are the valuable assets of a child. They play a vital role for mastication, phonetics, esthetics and social acceptance. Nowadays pulp therapy of primary teeth solves the problem of space loss, maintains the integrity of jaw growth and assimilation ${ }^{(1)}$.

Pulp therapy of primary teeth with necrotic pulp constitutes a great difficulty to pediatric dentists due to complex anatomy of the canal system which

\footnotetext{
* Dentist at Ministry of Health, B.D.S, Faculty of Dentistry, October University for Modern Sciences and Arts ** Professor in Pediatric Dentistry and Dental Public Health Department, Faculty of Dentistry, Ain Shams University. *** Lecturer in Pediatric and Community Dentistry Department, Faculty of Dentistry, Minia University.
} 
make microorganisms proliferate and invade lateral canals, apical deltas and dentinal tubules ${ }^{(2)}$.

Different microorganisms were isolated from necrotic primary teeth as Enterococcus faecalis, Staphylococcus aureus, Bacteroid fragilis, blackpigmented bacilli, Mutans streptococci, Candida and another anaerobic species ${ }^{(3)}$.

One of the critical goals of pulp therapy in deciduous teeth is the elimination of microorganisms which are the main cause of peri-radicular lesions. Although removal of infected pulp tissue decreases the number of microorganisms, an obturating material with strong antibacterial potential is essential to enhance disinfection of pulp space procedures improving the prognosis of pulpectomy ${ }^{(4)}$.

Various obturation materials have been used for the obturation of primary teeth and ZOE was the traditional obturation material. Also, calcium hydroxide and iodoform based materials have gained wide popularity. Another commercially available product is Endoflas which is a mixture of ZOE, calcium hydroxide and iodoform is ${ }^{(5)}$.

Therefore, the current study was conducted to compare the antibacterial potential of some of the commonly used obturation materials for primary teeth; ZOE only, calcium hydroxide with iodoform (Metapex) and a mixture of zinc oxide eugenol, calcium hydroxide and iodoform (Endoflas) against selected strains of bacteria; Enterococcus faecalis and Bacteroid fragilis.

\section{MATERIALS AND METHODS}

Agar diffusion method was used ${ }^{(6)}$ in which the antibacterial potential of ZOE (Septodont, India), Calcium hydroxide and Iodoform (Metapex, Korea) and Endoflas (Sanlor Lab, Colombia) against Enterococcus faecalis and Bacteroid fragilis was tested by measuring the inhibition zone around each material. Procedures were carried out following the guidelines of NCCLS (2010) ${ }^{(7)}$.

\section{Purification and incubation of bacterial cul-} ture: The standard bacterial strains of $E$. faecalis and B. Fragilis were obtained from microbiology department, El-Azhar University in Cairo. A single colony of each bacteria was picked with a sterile swab and transferred into fluid broth medium (Oxoid, UK), then aerobically incubated at $37^{\circ} \mathrm{C}$. The density of the organism suspensions was adjusted by spectrophotometer to contain $10^{8} \mathrm{cfu} / \mathrm{ml}$.

Preparation of culture medium: Mueller Hinton agar was prepared then sterilized by autoclaving. The medium was cooled down to the room temperature. Finally, it was poured in 18 petri dishes ( 9 for each type of bacteria).

Antimicrobial Susceptibility Test (Screening test by agar diffusion method): The inoculum was inoculated into Mueller- Hinton agar petri dishes using sterile swab and a fresh mix of each obturating material is loaded in the agar plates. Around each tested obturating material, the inhibition zone was measured after 24 hours at $37 \mathrm{Co}$.

\section{Statistical analysis:}

The mean values of inhibition zone around each tested material and standard deviation were calculated. Kolmogorov-Smirnov and Shapiro-Wilk tests were used to test normality. Data followed a normal distribution. One-way ANOVA followed by Tukey post hoc test were used to compare between more than two groups $(\mathrm{p}<0.05)$. Statistical analysis was performed with IBM® ${ }^{\circledR}$ SPSS ${ }^{\circledR}$ Statistics Version 20 for Windows.

\section{RESULTS}

\section{1- Bacteroid fragilis}

ZOE displayed a statistically significant difference with each of Endoflas and Metapex $(\mathrm{p}<0.001)$. Also, a statistically significant difference was found between (Endoflas) and (Metapex) where $(\mathrm{p}<0.001)$ as shown in table $(1)$. 
Endoflas showed the highest mean value of inhibition zone followed by ZOE, while the least mean value of inhibition zone was found in Metapex (figures $1 \& 3$ ). There was a statistically significant difference between ZOE, Endoflas and Metapex $(p<0.001)$.

\section{2- Enterococcus faecalis:}

Again, ZOE showed a statistically significant difference with each of Endoflas $(\mathrm{p}<0.001)$ and

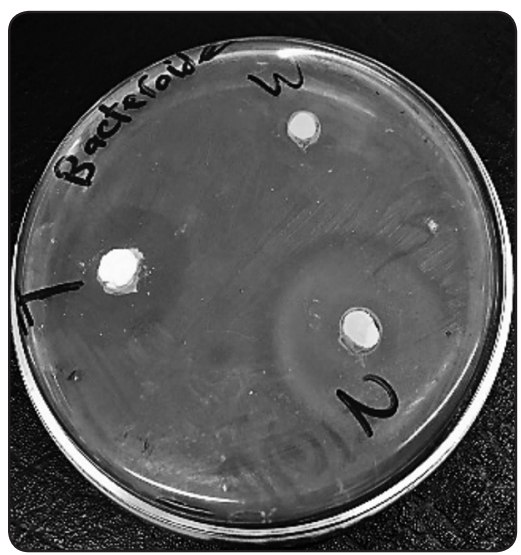

Fig. (1): Antimicrobial susceptibility testing for the three obturating materials by agar diffusion method against B. fragilis. Obturating materials numbering were; (1) ZOE; (2) Endoflas, and (3) Metapex.
Metapex $(\mathrm{p}=0.039)$. The difference between Endoflas and Metapex was statistically significant $(\mathrm{p}<0.001)$ (table 1).

Endoflas showed the highest mean value of inhibition zone followed by (Metapex), while the least mean value of inhibition zone was found in ZOE (figures 2 and 3). There was a statistically significant difference between ZOE, Endoflas and Metapex $(p<0.001)$.

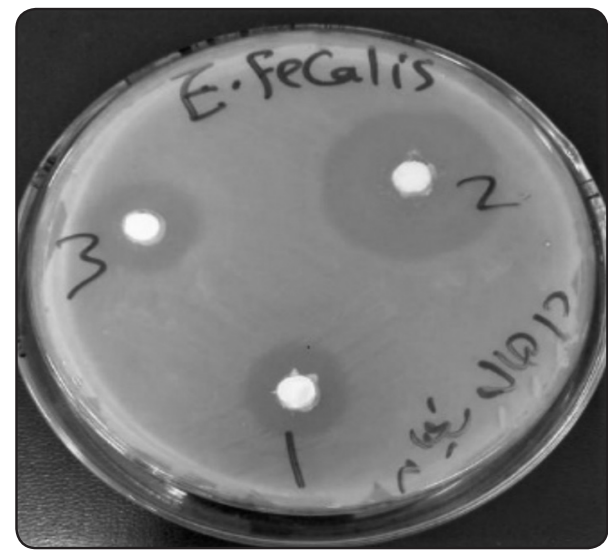

Fig. (2): Antimicrobial susceptibility testing for the three obturating materials by agar diffusion method against E. faecalis. Obturating materials numbering were; (1) ZOE; (2) Endoflas, and (3) Metapex.

TABLE (1) Mean and standard deviation (SD) values of inhibition zone of different groups.

\begin{tabular}{|c|c|c|c|c|}
\hline \multirow{3}{*}{ Variable } & \multicolumn{4}{|c|}{ Inhibition zone } \\
\hline & \multicolumn{2}{|c|}{ Bacteroid fragilis } & \multicolumn{2}{|c|}{ Enterococcus faecalis } \\
\hline & Mean & SD & Mean & SD \\
\hline Zinc-oxide and Eugenol & $22.33^{b}$ & 0.58 & $15.00^{\mathrm{c}}$ & 1.00 \\
\hline Endoflas & $32.33^{\mathrm{a}}$ & 2.52 & $29.33^{\mathrm{a}}$ & 1.15 \\
\hline Metapex & $11.00^{\mathrm{c}}$ & 1.00 & $18.33^{b}$ & 1.53 \\
\hline p value & \multicolumn{2}{|c|}{$<0.001 *$} & \multicolumn{2}{|c|}{$<0.001 *$} \\
\hline
\end{tabular}

Small letters in the same column indicate significant difference between means,

*; significant $(p<0.05) n s ;$ non-significant 


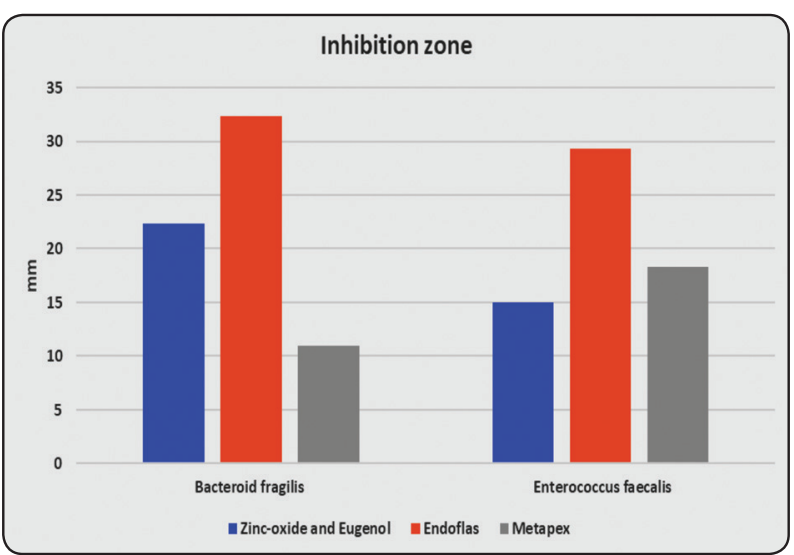

Fig. (3): Bar chart representing inhibition zone for different group.

\section{DISCUSSION}

Pulp therapy of infected primary teeth is a complicated issue for pediatric dentists. The tortuous and complex canal system make mechanical preparation and irrigation of canals not enough for complete removal of entrapped microorganisms. Hence, an obturation material with strong antibacterial effect is essential for the success of pulpectomy ${ }^{\left({ }^{8}\right)}$.

Various obturating materials for root canals of primary teeth have been tried in pediatric dentistry. In the current study, ZOE, Metapex and Endoflas were tested since they are the most widely used ones ${ }^{(5)}$.

Despite that many bacterial species could be detected in necrotic pulp of the primary teeth, the microbiology of most pyogenic dental infections contains B. fragilis and E. faecalis as the most dominant microorganisms. Moreover, B. fragilis and E. faecalis are reported as therapy-resistant bacteria in the infected pulp microenvironment showing resistance to penicillin treatment ${ }^{(9,10)}$.

In the present study, the antimicrobial susceptibility test was performed by the agar diffusion method due to the merits of this technique as it is cheap, available, fast technique that allows visibility of bacterial growth and results are accurate and available after short time ${ }^{(11)}$.

Regarding the growth of $\mathrm{B}$. fragilis, this study revealed that the three obturating materials reported antibacterial effect, however, Endoflas had the highest antimicrobial potential followed by ZOE and finally Metapex $(\mathrm{p}<0.001)$. Parallel results were reported by Ruchi et al. (2014) ${ }^{(12)}$, Bhargava et al. (2015) ${ }^{(13)}$ and Navit et al. (2016) ${ }^{(14)}$. On the other hand, Hegde et al. (2012) ${ }^{(15)}$ demonstrated that ZOE had higher efficacy followed by Endoflas and Katerine et al. (2017) ${ }^{(16)}$ reported that the pure Iodoform paste and its mixture with calcium hydroxide were the most effective obturating materials.

Several factors could be responsible for this dissimilarity in results such as heterogeneity of the tested species, and difference in techniques used for detection of the antibacterial effect with different sensitivities. Katerine et al. (2017) ${ }^{(16)}$ used mixedspecies biofilm that was studied by using confocal laser scanning microscopy.

Regarding the inhibition of growth of E. faecalis, Endoflas was the superior followed by Metapex and ZOE was least effective. This goes in accordance with Reddy et al. (2007) ${ }^{(17)}$.

Moreover, Ibrahim et al. (2017) ${ }^{(18)}$ concluded that Endoflas had the highest antimicrobial effect against E. faecalis while calcium hydroxide and iodoform paste was least effective. This difference in the antimicrobial effect of the obturation materials may be due to the difference in concentration of the chemical substances like the concentration of eugenol ${ }^{(18)}$.

It is noteworthy to mention that Iodoform lack the antimicrobial activity in vitro but it in vivo results are divergent, suggesting that its function may be helped by stimulating the biological body reaction ${ }^{(19)}$. 


\section{CONCLUSION}

1. The three tested obturation materials reported antibacterial effect.

2. Endoflas was superior to Metapex and ZOE and the difference was statistically significant.

\section{REFERENCES}

1. Hashemi, Z., Manzuri, L., Rahimi, F., Farzadmoghadam, M., \& Gholami, S. A. S. Awareness of care and importance of primary teeth among mothers in Yasuj 2017. Caspian Journal of Dental Research, 2019; 8(1), 24-30.

2. Öter, B., Topcuoglu, N., Tank, M. K., \& Cehreli, S. B. Evaluation of antibacterial efficiency of different root canal disinfection techniques in primary teeth. Photomedicine and laser surgery, 2018; 36(4), 179-184.

3. Chavez de Paz LE, Dahlen G, Molander A, Moller A and Bergenholtz G. Bacteria recovered from teeth with apical periodontitis after antimicrobial endodontic treatment. Int Endod J. 2003; 36:500-508.

4. Sapna, H., Priti Kamlesh, L., Dinesh Rao, B., \& Shubha, A. B. An in vitro evaluation of antimicrobial efficacy of primary root canal filling materials. Journal of Clinical Pediatric Dentistry,2012; 37(1), 59-64.

5. Saltzman B, Sigal M, Clokie C, Rukavina J, Titley K, Kulkarni GV. Assessment of a novel alternative to conventional formocresol-zinc oxide eugenol pulpotomy for the treatment of pulpally involved human primary teeth: diode laser-mineral trioxide aggregate pulpotomy. Int J Paediatr Dent. 2005 Nov; 15(6):437-447.

6. Hindler ,J.A.;Howard,B.J. and Keiser, J.F.Antimicrobial agents and Susceptibility testing. In: Howard BJ(Editor), Clinical and pathogenic Microbiology. Mosby-Year Book Inc., St. Louis, MO,USA,.1994.

7. National Committee for Clinical and Laboratory Standards guidelines (NCCLS). Performance standards for Antimicrobial susceptibility testing, twentieth informational supplement,. 2010.

8. American Academy of Pediatric Dentistry. Guideline on pulp therapy for primary and young permanent teeth. Pediatric Dentistry.2009;31(6):179
9. Kannangara, D. W., Thadepalli, H., \& McQuirter, J. L. Bacteriology and treatment of dental infections. Oral Surgery, Oral Medicine, Oral Pathology.1980; 50(2), 103-109.

10. Da Silva LA, Nelson-Filho P, Faria G, Souza-Gugelmin MC, Ito IY. Bacterial profile in primary teeth with necrotic pulp and periapical lesions. Braz Dent J. 2006; 17(2): 144-148.

11. Serisier DJ, Tuck A, Matley D, Carroll MP, Jones G,Eur J Clin. Antimicrobial susceptibility and synergy studies of cystic fibrosis sputum by direct sputum sensitivity testing, Microbiol Infect Dis. 2012 Nov; 31(11):3211-3216.

12. Ruchi A, Parul R, Deepak P. Bhayya. A Comparative Evaluation of Antimicrobial Efficacy of Three Endodontic Sealers. IOSR-JDMS. 2014; 3: 90-93.

13. Bhargava R, Shashikala K, Bhargava R. Antibacterial efficacy of three root canal sealers against Enterococcus faecalis and Candida albicans: An in vitro study. SRM J Res Dent Sci.2015; 6:170-4. 23.

14. Navit S, Neha J, Suleman AK, Seema M, Anshul S, et al. Antimicrobial efficacy of Contemporary Obturating materials used in Primary teeth. An vitro study: J Clin Diagn Res. 2016; 10: 9-12.

15. Hegde S, Priti K, Dinesh RB, Shubha AB. An in vitro Evaluation of Antimicrobial Efficacy of Primary Root Canal Filling Materials. J Clin Pediatr Dent. 2012; 37:59-64.

16. Katerine JP, Ceci NC, Ana RR Renata DM , Ya Shen, Markus H, Fernanda GP. Antibiofilm Activity of Five Different Endodontic Filling Materials Used in Primary Teeth Using Confocal Laser Scanning Microscopy. Pediatr Dent. 2017; 39:145-149.

17. Reddy S, Ramakrishna Y. Evaluation of Antimicrobial Efficacy of Various Root Canal Filling Materials Used in Primary Teeth: A Microbiological Study. J Clin Pediatr Dent. 2007; 31:195-199.

18. Ibrahim, I. A., Masallat, D. T., Ibrahim, A. H., \& El Kalla, I. H. Antimicrobial Activities of Commercially Available Obturating Materials in Primary Teeth. Egyptian Journal of Medical Microbiology.2019; 28(2):77-84.

19. Estrela C, Estrela CR, Hollanda AC, Decurcio Dde A, Pécora JD. Influence of iodoform on antimicrobial potential of calcium hydroxide. J Appl Oral Sci. 2006; 14(1):33-37. 